\title{
Clinical outcomes following treatment of peri-implantitis at formerly grafted and non-grafted implant sites
}

IMPLANT THERAPY OUTCOMES, SURGICAL ASPECTS
Ausra Ramanauskaite, Kathrin Becker, Prof. Frank Schwarz

Department of Oral Surgery, Heinrich Heine University Düsseldorf, Germany

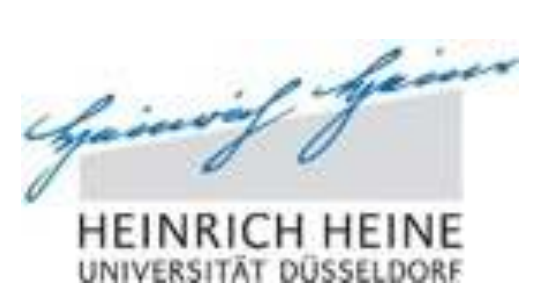

\section{Background}

Currently no evidence exists whether the effectiveness of treatment of peri-implantitis is influenced by initial bone grafting procedures.

\section{The aim}

This retrospective analysis aimed at comparing the clinical outcomes following nonsurgical and surgical therapy of peri-implantitis at formerly grafted and non-grafted (i.e. pristine) implant sites.

\section{Materials \& Methods}

A total of 29 patients diagnosed with peri-implantitis at formerly grafted and non-grafted sites (Fig. 1) were included. Each subject had received either nonsurgical or combined surgical treatment procedures at respective implants. The primary outcome variable was disease resolution (i.e. absence of BOP (bleeding on probing) and probing pocket depths (PD) $\geq 6 \mathrm{~mm}$ at peri-implantitis sites). Reduction of mean BOP and maximum $P D$ values were defined as secondary outcome variables. The mean follow-up period was $176.75 \pm 135$ weeks.

Figure 1. Flow-chart presenting four patient groups.

\section{9 patients / 46 implants}

\section{Implants placed in}

non-grafted (pristine) bone

$\mathrm{n}=19$ implants,

14 patients

treatment*

= 4 implants

2 patients
Nonsurgical

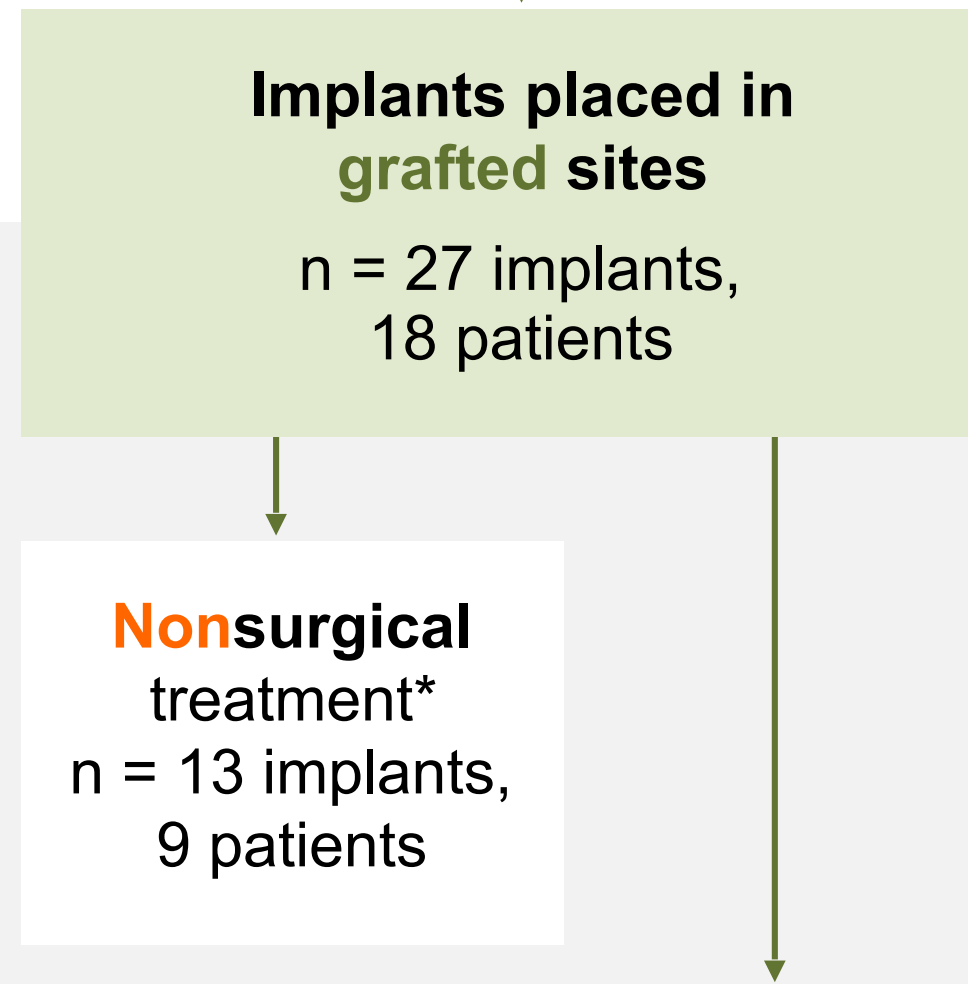

Surgical treatment* $\mathrm{n}=14$ implants 9 patients
Surgical treatment** $\mathrm{n}=15$ implants 12 patients
Reduction of mean BOP (\%).

The differences among the groups did not yield a significant difference (patient leve $p=0.575$, implant level $p=0.316$, Kruskal Wallis test .

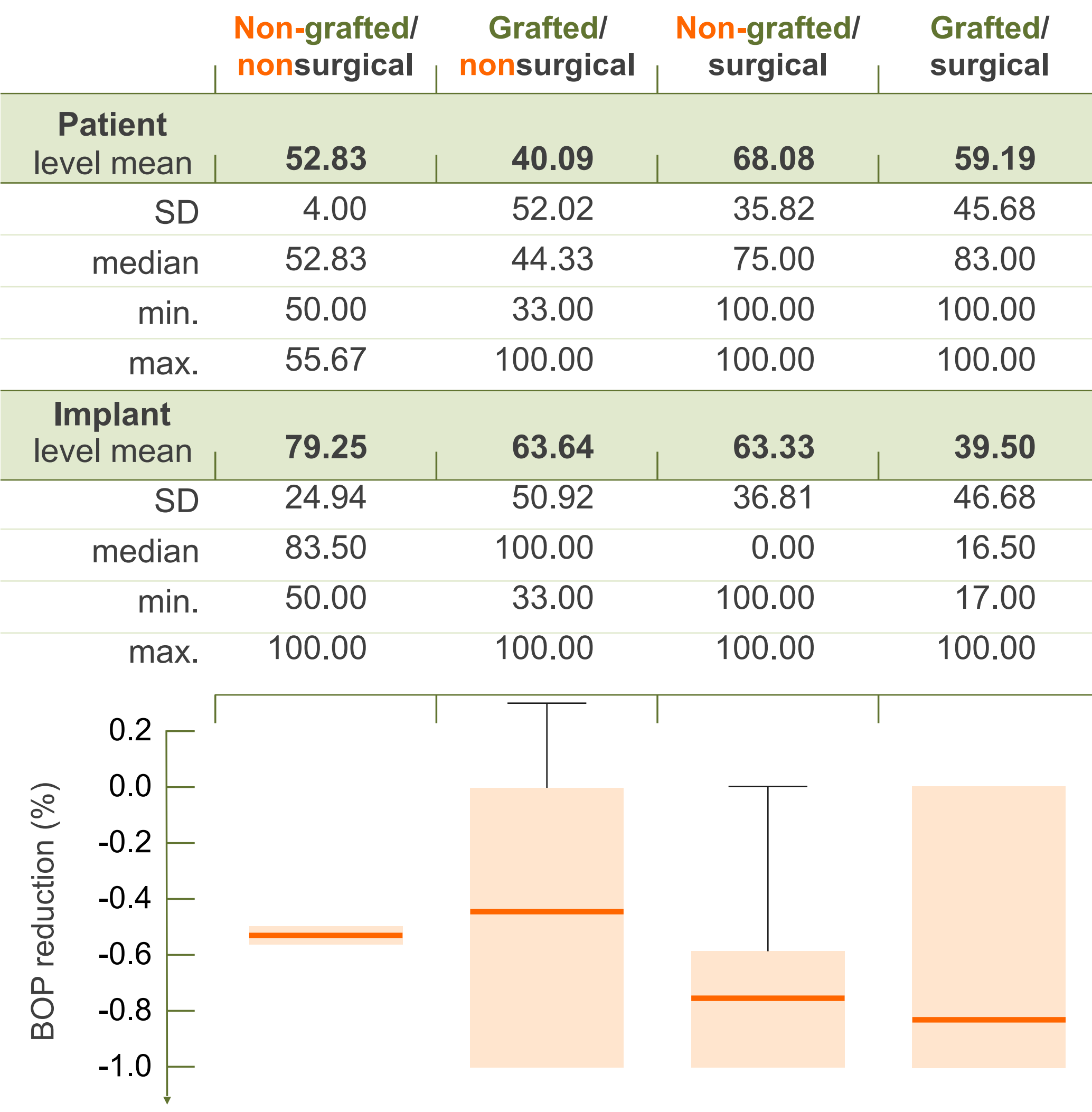

Box plot presenting mean BOP reduction among the four patient groups with no significant difference $(p=0.575)$.

Recudtion of maximum PD ( $\mathrm{mm}$ ).

No significant difference among the groups was observed (patient level $p=0.728$, implant level $p=0.263$, Kruskal Wallis test)

\begin{tabular}{rrrrrr} 
& $\begin{array}{c}\text { Non-grafted/ } \\
\text { nonsurgical }\end{array}$ & $\begin{array}{c}\text { Grafted/ } \\
\text { nonsurgical }\end{array}$ & $\begin{array}{c}\text { Non-grafted/ } \\
\text { surgical }\end{array}$ & $\begin{array}{c}\text { Grafted/ } \\
\text { surgical }\end{array}$ \\
\hline $\begin{array}{r}\text { Patient } \\
\text { level mean }\end{array}$ & -0.33 & -1.24 & -2.00 & -0.91 \\
\hline SD & 2.36 & 2.08 & 3.14 & 1.47 \\
\hline median & -0.33 & 0.00 & -2.00 & -1.00 \\
\hline min. & -2.00 & -6.00 & -6.00 & -3.50 \\
\hline max. & 1.33 & 0.33 & 4.00 & 1.00 \\
\hline Implant & & & & \\
level mean & -2.00 & -1.91 & -1.53 & -0.25 \\
\hline SD & 1.50 & 1.81 & 3.42 & 1.91 \\
\hline median & -2.00 & -2.00 & 0.00 & 0.00 \\
min. & -2.00 & -6.00 & -6.00 & -4.00 \\
max. & 1.00 & 0.00 & 4.00 & 4.00 \\
& & & &
\end{tabular}

Nonsurgical treatment *

(i.e. mechanical, Er:YAG laser or photodynamic therapy (PDT))

Surgical combined treatment **

(i.e. implantoplasty $+/$ - augmentative therapy)

\section{Results}

Disease resolution among the four groups.

\begin{tabular}{c|c|c|c|c|c} 
& $\begin{array}{c}\text { Non- } \\
\text { grafted/ } \\
\text { non- } \\
\text { surgical }\end{array}$ & $\begin{array}{c}\text { Grafted/ } \\
\text { non- } \\
\text { surgical }\end{array}$ & $\begin{array}{c}\text { Non- } \\
\text { grafted/ } \\
\text { surgical }\end{array}$ & $\begin{array}{c}\text { Grafted/ } \\
\text { surgical }\end{array}$ & Total \\
\hline $\begin{array}{c}\text { Patient } \\
\text { level }\end{array}$ & $\begin{array}{c}2 / 2 \\
(100 \%)\end{array}$ & $\begin{array}{c}6 / 9 \\
(67 \%)\end{array}$ & $\begin{array}{c}7 / 12 \\
(58 \%)\end{array}$ & $\begin{array}{c}5 / 9 \\
(56 \%)\end{array}$ & $\begin{array}{c}20 / 32 \\
(62.5 \%)\end{array}$ \\
\hline $\begin{array}{c}\text { Implant } \\
\text { level }\end{array}$ & $4 / 4$ & $10 / 13$ & $9 / 15$ & $7 / 14$ & $30 / 46$ \\
\hline
\end{tabular}

No significant difference among the groups was found (patient level $p=0.673$, implant level $p=0.21$, Chi-square test)

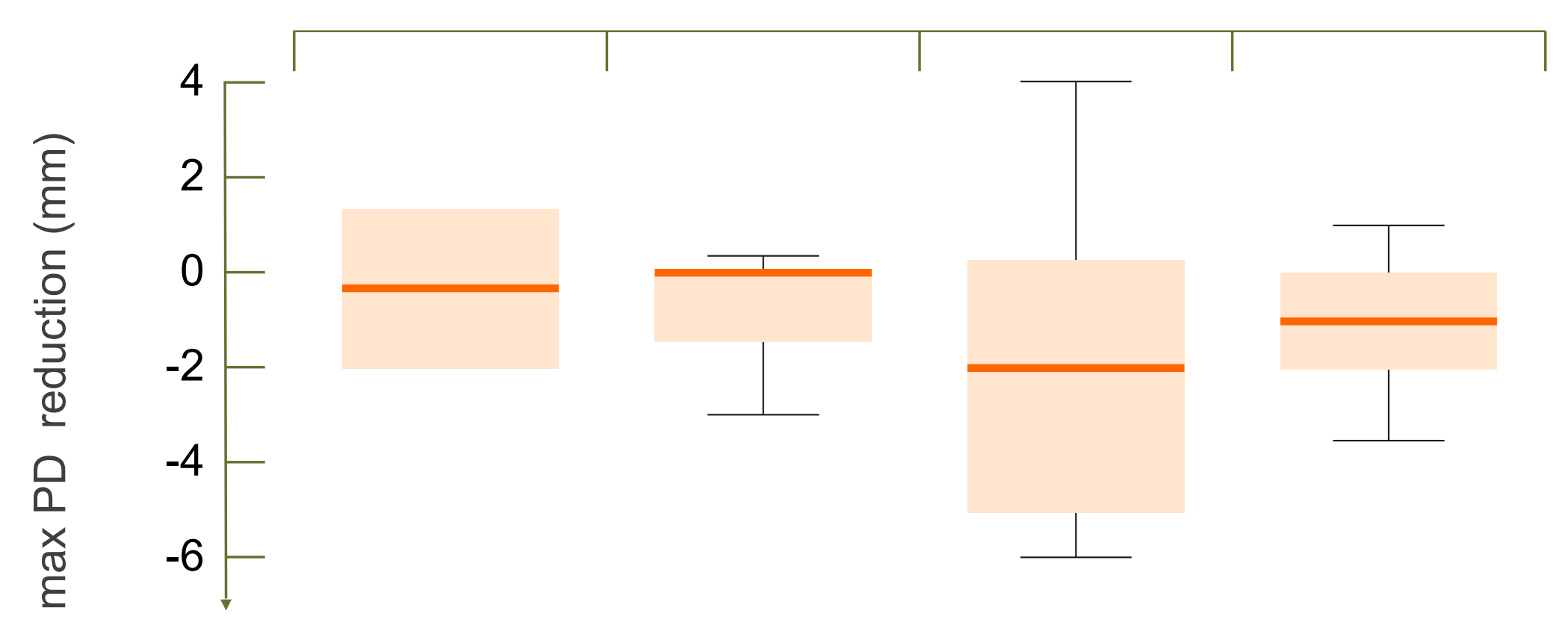

Box plot illustrating maximum PD reduction among the four patient groups that did not reach a significant difference $(p=0.728)$.

\section{Conclusions}

The effectiveness of nonsurgical and surgical treatment of peri-implantitis was comparable at both formerly grafted and non-grafted implant sites.

Corresponding author: Ausra Ramanauskaite Ausra.Ramanauskaite@med.uni-duesseldorf.de 